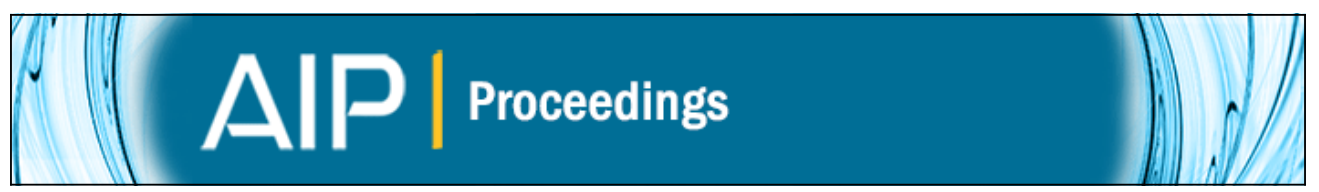

The Impact of Cold Dark Matter Variants on the Halos of the First Stars and Galaxies: Angular Momentum and Vortex Creation in BEC Dark Matter

Tanja Rindler-Daller and Paul R. Shapiro

Citation: AIP Conference Proceedings 1294, 274 (2010); doi: 10.1063/1.3518874

View online: http://dx.doi.org/10.1063/1.3518874

View Table of Contents:

http://scitation.aip.org/content/aip/proceeding/aipcp/1294?ver=pdfcov

Published by the AIP Publishing

Articles you may be interested in

Statistical properties of dark matter mini-halos

AIP Conf. Proc. 1480, 409 (2012); 10.1063/1.4754403

First stars and WIMP dark matter: A state of the art review

AIP Conf. Proc. 1480, 71 (2012); 10.1063/1.4754331

Collision of BEC dark matter structures and comparison with the collision of ideal gas structures

AIP Conf. Proc. 1318, 90 (2010); 10.1063/1.3531645

Dark Stars: the First Stars in the Universe may be powered by Dark Matter Heating AIP Conf. Proc. 1166, 33 (2009); 10.1063/1.3232192

The Effect of Dark Matter on the First Stars: A New Phase of Stellar Evolution AIP Conf. Proc. 990, 42 (2008); 10.1063/1.2905656 


\title{
The Impact of Cold Dark Matter Variants on the Halos of the First Stars and Galaxies: Angular Momentum and Vortex Creation in BEC Dark Matter
}

\author{
Tanja Rindler-Daller, ${ }^{* \dagger}$ and Paul R. Shapiro* \\ ${ }^{*}$ Department of Astronomy and Texas Cosmology Center, The University of Texas at Austin, USA \\ ${ }^{\dagger}$ Institut für Theoretische Physik, Universität zu Köln, Cologne, Germany
}

\begin{abstract}
If cold dark matter elementary particles form a Bose-Einstein condensate, their superfluidity may distinguish them from other forms of cold dark matter, including the creation of quantum vortices. We have shown that such vortices are favored in strongly-coupled condensates. Vortex creation causes central densities to drop, thus affecting the dynamics of the gaseous baryonic component and subsequently star formation.
\end{abstract}

Keywords: cold dark matter; Bose-Einstein condensation; large-scale structure PACS: $95.35 .+\mathrm{d} ; 67.85 . \mathrm{Jk} ; 98.80 .-\mathrm{k}$

The formation of the first stars depended upon the internal structure at the center of their host dark matter halos, where baryonic cooling and condensation occured first. While the halos of collisionless cold dark matter (CDM) are characterized by the singular NFW density profile, other forms of cold dark matter have been proposed which alter flatten the central profile. Among these are light, self-interacting bosonic particles that form a Bose-Einstein condensate (BEC). We shall explore one aspect of BECs on the structure of the halos hosting the first stars: the effects of the angular momentum that results from tidal torquing during large-scale structure formation (see also [2]).

We describe self-gravitating BEC halos with varying degrees of rotational support by self-consistently coupling the Gross-Pitaevskii equation of motion for the complex macroscopic BEC wave function to the Poisson equation. For simplicity, we consider incompressible, uniformly-rotating spheroids, which are approximate solutions of that system of equations. This family of solutions is characterized by the angular rotation $\Omega$ or, equivalently, the eccentricity $e$. We use an energy argument to derive the critical angular velocity $\Omega_{c}$ for the creation of a singly-quantized, straight vortex in a rotating, self-gravitating BEC halo by finding the angular velocity which lowers the total energy in the presence of that vortex. In general, we must determine what set of BEC parameters, namely particle mass $m$ and self-interaction coupling $g$, makes the spheroid rotation velocity for a given $e$ equal the critical value above which the vortex forms, $\Omega=\Omega_{c}$.

The $e$-values of interest for $\mathrm{BEC} / \mathrm{CDM}$ halos are those which correspond to the degree of halo rotational support found by cosmological N-body simulations of the CDM universe, as expressed by the ratio $\lambda=L \sqrt{|W|} /\left(G M^{5 / 2}\right): \lambda$ has values in the range $[0.01,0.1]$ with a median of 0.05 [1]. For our spheroids, $\lambda$ can be expressed in terms of $e$ only (see [2]). The values $\lambda=(0.01,0.05,0.1)$ correspond to $e=(0.051,0.249,0.464)$, 


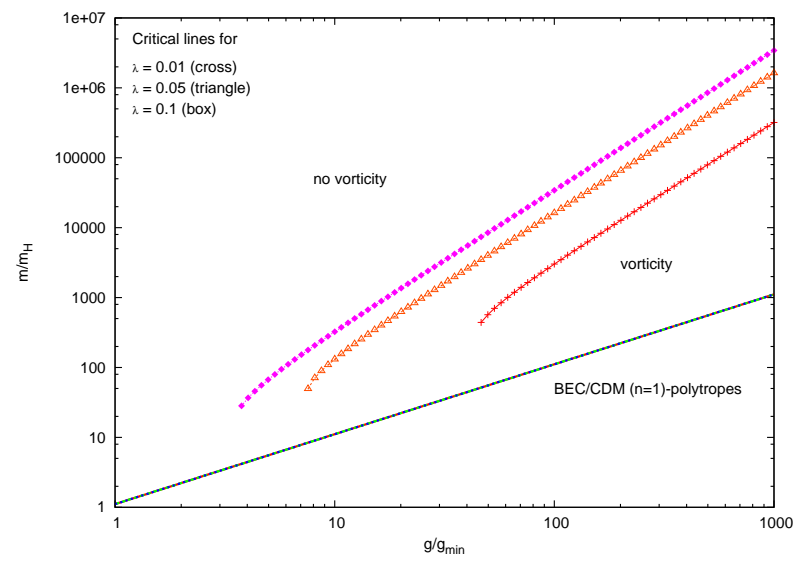

FIGURE 1. Dimensionless BEC particle mass $m / m_{H}$ vs. coupling strength $g / g_{\text {min }}$ : critical lines $\Omega=\Omega_{c}$ (top curves) and BEC/CDM (n=1)-polytropes for the same $e$-values (bottom curve). $m_{H}$ and $g_{\min }$ depend only on the halo parameters $a$ (size), mean density $\rho, m_{H} \equiv \hbar /\left(a^{2} \sqrt{\pi G \rho}\right)$ and $g_{\min } \equiv \hbar^{2} /\left(2 \rho a^{2}\right)$, e.g. for a Milky-Way-sized halo, $m_{H} \sim 10^{-58} \mathrm{~g}$ and $g_{\min } \sim 10^{-76} \mathrm{erg} \mathrm{cm}^{3}$.

respectively. The critical lines are shown in Figure 1 such that the CDM halo rotation velocity $\Omega$ for those $e$-values equals the critical value $\Omega_{c}$ above which a vortex forms. For each critical line, no vortex is allowed for parameters in the space above the line (higher $\lambda \rightarrow$ higher curves).

In the strongly-coupled regime, non-rotating BEC halos in gravitational equilibrium are $(n=1)$-polytropes (see e.g. [3], [4]), which is approximately valid, too, for CDM halo $\lambda$-values. The resulting relationship between $\mathrm{m} / \mathrm{m}_{H}$ and $\mathrm{g} / \mathrm{g}_{\min }$ is plotted in Figure 1 as well. Since the BEC parameters which satisfy that relationship are all well below our critical curves for which $\Omega=\Omega_{c}$, BEC/CDM halos, in general, will typically form vortices. However, vortex creation causes the central halo density to drop. If BEC halos form with substantially lower central densities than do standard CDM halos, the ability of baryons there to radiatively cool and condense to become self-gravitating, a precondition for star formation, will be suppressed.

\section{ACKNOWLEDGMENTS}

This work was supported by the DFG under research unit FG 960, by NSF grant AST 0708176, by NASA grant NNX07AH09G and by Chandra grant SAO TM8-9009X.

\section{REFERENCES}

1. J. Barnes, and G. Efstathiou, ApJ 319, 575 (1987)

2. T. Rindler-Daller, and P. R. Shapiro, arXiv:0912.2897 (2009), to appear in Bash Symp.09, Proc.ASP

3. J. Goodman, New Astronomy 5, no.2, 103 (2000)

4. C.G. Böhmer, and T. Harko, JCAP 06, 025 (2007) 